\title{
Origin of methyl torsional potential barrier - An overview
}

\author{
TAPANENDU KUNDU*, BISWAJIT PRADHAN and BHANU P SINGH \\ Department of Physics, Indian Institute of Technology - Bombay, Powai, \\ Mumbai 400 076, India \\ e-mail: tkundu@phy.iitb.ac.in
}

\begin{abstract}
This paper presents the evolution of views on methyl internal rotation potential barrier. Various mechanisms proposed for the origin of torsional barrier in ethane have been reviewed. Inadequacy of one dimensional description of internal rotation has been highlighted in small methyl conjugated molecules in the light of its multidimensional nature. The effect of skeletal flexing on the picture of barrier formation by dissecting the barrier energy into potential type, virial type and symmetry type is described. The role of $\pi$ and $\sigma$ electrons at different stages of molecular flexing is discussed. The analysis identifies the dominant contributions to barrier origin as $\pi$-bonding changes during rigid rotation and $\sigma$-bonding changes resulting from bond lengthening during methyl group rotation. The contribution of lone pair electrons in determining the preferred structure of the methyl group in imine compounds such as 1-methyl $2-(1 \mathrm{H})$-pyridinimine is presented.
\end{abstract}

Keywords. Methyl torsion; methyl torsional barrier; large amplitude motion.

\section{Introduction}

In methylated molecules, the methyl group can rotate around a single bond so that during a full $360^{\circ}$ rotation it thrice encounters an unstable and a relatively stable conformation. The energy difference between these two conformers gives rise to potential barrier to this rotation. The quantum mechanical nature of this hindered rotation in molecules was recognized in the early years of quantum theory. ${ }^{1}$ Since then, detailed experimental and theoretical studies have been attempted to establish the nature of this barrier. Recent interest in this torsional potential barrier has grown tremendously due to accurate predictions of molecular geometry enabled by large scale $a b$ initio calculations and precise potential shape information made available by high resolution FTIR, microwave and supersonic jet spectroscopies. Rydberg state jet spectroscopy ${ }^{2}$ has also been exploited for probing ground state modes as well as those for the Rydberg states of the molecules due to increased clarity and narrow lines. The barrier height varies from a few wave number as in toluene to a thousand wave number as in ethane. It is thus highly sensitive to the local chemical structure of the molecule. Insight into the origin of this barrier is in a way fundamental to the understanding of the preferred structural orientation of the methyl group and the internal dynamics in flexible molecules accompanying methyl rotation. These studies assume special significance in the realm of stereochemistry for engineering the molecular functionality. It may also facilitate the deeper insight into various biomolecular processes.

\footnotetext{
*For correspondence
} 
In this paper, we review the evolution of the understanding of the origin of methyl torsional potential barrier for some key molecules. We begin with ethane molecule, a simple textbook example and follow it with small methyl conjugated organic molecules. We will concentrate on two isoelectronic systems, acetaldehyde and propene, long regarded as benchmark molecules where the methyl group is connected with vicinal unsaturated polar double bond in the former and that with nonpolar double bond in the later. At the end, we will discuss few aromatic systems of special interest. Here, we have not attempted to provide an exhaustive coverage of the extensive literature on internal rotation. We refer the reader to Lister, MacDonald and Owen's monograph ${ }^{3}$ and Reed and Weinhold's review ${ }^{4}$ for basic references. Payne et $a l^{5}$ and Villard et $a l^{6}$ provide in-depth review of $a b$ initio barrier calculations. Nature of methyl rotation in aromatic compounds has been review by Ito. ${ }^{7 \mathrm{a}}$ Spangler and Pratt ${ }^{7 \mathrm{~b}}$ have focused on internal rotation dynamics in their recent article.

\section{Theoretical approach to methyl rotation}

In this paper, we have concentrated mainly on the conceptual aspects of the formation of torsional barrier from the theoretical point of view. In this regard $a b$ initio calculations have been quite successful in simulating the barrier energy for internal rotation due to accurate predictions of molecular geometry. The barrier is the difference in total electronic energy between the equilibrium and the maximum energy conformer during the methyl rotation. Detailed information on different interactions during methyl rotation can be obtained by partitioning the overall energy terms into differences in attractive electron-nuclear $\left(V_{n e}\right)$, repulsive nuclear-nuclear $\left(V_{n n}\right)$ and electron-electron $\left(V_{e e}\right)$ interactions and kinetic energies $(\Delta T)$. In this scheme, the barrier energy,

$$
\Delta E=\Delta T+\Delta V_{e e}+\Delta V_{n n}+\Delta V_{n e} .
$$

The attractive and repulsive contributions to the total potential energy change are $\Delta V_{a} \equiv \Delta V_{n e}$ and $\Delta V_{r} \equiv \Delta V_{e e}+\Delta V_{n n}$ respectively.

From an energetic viewpoint, the internal rotation potential function $V(\tau)$, where $\tau$ is the torsional coordinate, can be thought of as the one dimensional representation of the molecular potential surface passing through the minimum energy pathway with respect to all other degrees of freedom. The traditional approach is to expand the potential function $V(\tau)$ in a Fourier series in $\tau$,

$$
V(\tau)=\frac{1}{2} \sum_{n} V_{n}(1-\cos n \tau) .
$$

Because of the 3-fold symmetry of the methyl group which reproduces itself through cyclic permutation of hydrogens, the only non-zero coefficients are $V_{3}, V_{6}$ etc. Thus, for methyl torsional potential,

$$
V(\tau)=\frac{V_{3}}{2}(1-\cos 3 \tau)+\frac{V_{6}}{2}(1-\cos 6 \tau)+\cdots
$$


The odd terms affect both the barrier height and shape. Amongst these, $V_{3}$ is the most important higher determinant whereas $V_{6}$ affects only the shape of the barrier. Torsional energy levels are derived from pure internal rotation Hamiltonian $H=F p^{2}+V(\tau)$, where $p$ is the momentum associated with methyl top rotation and $F$, the kinetic energy coefficient, ${ }^{8}$ relates to methyl rotor moment of inertia.

This one-dimensional description of internal rotation is the rigid rotation where the molecular geometry is fixed at its equilibrium configuration and only the torsional angle is allowed to vary and thus does not explicitly take into account interactions between torsion and other degrees of freedom. Due to this, rigid rotation fails to reproduce the three fold periodic potential.

From a Quantum Mechanical viewpoint, the minimum energy at any configuration should satisfy variational principle. The generalized virial theorem is $T=-E+$ $\sum_{\alpha} X_{\alpha} \cdot F_{\alpha}$ where, the nuclear virial $\sum_{\alpha} X_{\alpha} . F_{\alpha}$ is the contribution of nucleus $\alpha$ to the virial of the forces acting on electrons, $X_{\alpha}$ is the position vector of nucleus $\alpha$ and $F_{\alpha}$ is the net force acting on it. When the forces acting on the nuclei during rigid rotation vanish as they do at the variational minimum energy configuration, then $T=-E$. The importance of this generalized virial theorem is that it provides a tool for discussing internal rotation by identifying (i) different strain effects during internal rotation and (ii) the unrelaxed segment of the molecule that is responsible for that strain (see $\$ 3.2 b$ ). This, in a way, helps to create a dynamical picture of the methyl rotation.

\section{Discussion}

\subsection{Ethane}

In 1936, Kemp and Pitzer ${ }^{9}$ first recognized the hindered rotation in ethane from their original thermodynamic measurements. Despite the passage of 65 years, the underlying cause of this hindered rotation for this simple molecule remains unresolved. A number of views to explain the origin of the barrier to this rotation have been proposed, but none of them has been universally accepted.

In ethane, one of its two methyl groups rotates around the central carbon-carbon bond. Precise low temperature heat capacity measurement ${ }^{10}$ in 1951 has established the barrier to this rotation as $2.9 \mathrm{kcal} / \mathrm{mol}\left(\sim 1000 \mathrm{~cm}^{-1}\right)$. Several experimental ${ }^{9,10}$ and theoretical $^{4,11-14}$ studies confirm the preferred structure of ethane as staggered conformation (six hydrogen atoms are positioned as far apart as possible) whereas eclipsed one (the hydrogen atoms are in pairwise conjunction) is at the top of the barrier (figure 1). Late 1960s ideas ${ }^{11}$ on the origin of the barrier invoked Pauli-like exchange repulsion between $\mathrm{C}-\mathrm{H}$ bond orbitals. That is while ethane rotates towards eclipsed conformation, electrons in the $\mathrm{C}-\mathrm{H}$ bonds on two different carbon atoms experience repulsion which introduce the barrier. This is usually known as steric effect. In 1979, Brunck and Weinhold ${ }^{13}$ proposed that the cause of the barrier is due to the lengthening of the central $\mathrm{C}-\mathrm{C}$ bond involving vicinal interactions between six methyl $\sigma_{\mathrm{C}-\mathrm{H}}$ occupied bonding orbitals and associated $\sigma^{*}{ }_{\mathrm{C}-\mathrm{H}}$ antibonding orbitals. Bader $e t a l,{ }^{14}$ in 1990 , have offered a provocative explanation for the barrier in terms of the polarization of charge density in the $\mathrm{C}-\mathrm{C}$ bond. This originates from the change in molecular symmetry from $\mathrm{S}_{3}$ in staggered to $\mathrm{C}_{3}$ at the top of the barrier. This symmetry reduction induces quadrupole polarization of charge density in the electron cloud. It causes lengthening of the $\mathrm{C}-\mathrm{C}$ bond because this type of charge distribution is less effective in binding the carbon nuclei. In 1999, 

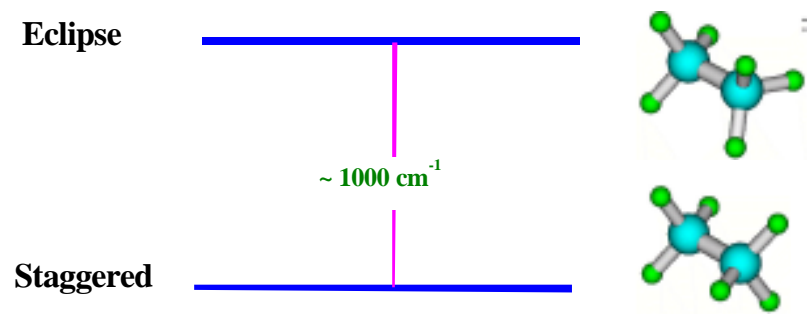

Figure 1. Ethane $\left(\mathrm{CH}_{3}-\mathrm{CH}_{3}\right)$ eclipsed (at the top of the barrier) and staggered (preferred structure) conformers.

Goodman et $\mathrm{l}^{15}$ argued that this $\mathrm{C}-\mathrm{C}$ bond weakening does not arise from the symmetry change accompanying ethane rotation. According to them, none of the above works has considered the effect of molecular relaxation on either Pauli repulsion or hyperconjugative charge transfer between $\sigma_{\mathrm{C}-\mathrm{H}}$ and $\sigma^{*} \mathrm{C}-\mathrm{H}$. In view of this, they have carried out a detailed flexing analysis in terms of structural, steric exchange and hyperconjugative charge transfer energy change during methyl rotation. The structural energy change represents the energy of the localized species defined by the Lewis structure, the steric exchange energy change represents the energy to preserve the mutual orthogonality of filled orbitals on atoms that are forced into spatial proximity by the rotation whereas the hyperconjugation interaction depends on the relative orientation of the donor acceptor type orbitals. This analysis shows that steric exchange repulsion interactions are not the main cause for ethane equilibrium geometry or $\mathrm{C}-\mathrm{C}$ bond lengthening. A detailed analysis of these energy changes in terms of the torsion angle and $\mathrm{C}-\mathrm{C}$ bond expansion concludes that the structural and hyperconjugative charge transfer are opposite to structural energy change in nature. These two form the barrier and the barrier energy can not be understood without taking into account the relaxation of $\mathrm{C}-\mathrm{C}$ bond. The recent paper in Nature by Pophristic and Goodman ${ }^{16}$ questioned the textbook explanation on ethane torsional barrier. To ascertain the exact cause of the barrier they have optimized the ethane structure by successive removal of different interactions from the calculations. The optimization without total exchange repulsion prefers staggered conformer. The removal of hyperconjugation from the calculation stabilizes the eclipsed structure. They concluded from this that hyperconjugation and not the steric repulsion is the origin of ethane internal rotation barrier.

\subsection{Small methyl conjugated molecules}

In molecules, the methyl group attached to an unsaturated vicinal bond stabilizes the eclipsed conformer where one of its $\mathrm{CH}$ bonds eclipses the unsaturated linkage and during its rotation encounters barrier (several hundreds of wave number) through staggered conformation obtained by $60^{\circ}$ rotation of methyl group. To visualise the origin of the barrier for these methyl conjugated systems we concentrate only on two basic molecules, acetaldehyde and propene. Both of them are isoelectronic, with identical numbers of $\pi$ and $\sigma$ electrons but differ in nature of the double bond. Acetaldehyde is having highly polar carbonyl $(\mathrm{C}=\mathrm{O})$ bond whereas it is nonpolar $\mathrm{C}=\mathrm{C}$ in propene. In this 
section we discuss different models to explain the potential barrier, its dependence on the nature of double bond and look into the physics behind the barrier from different perspectives.

Acetaldehyde and propene, simple conjugated molecules have had almost as much attention as ethane, long regarded as key molecules in understanding the energetics of internal rotation barriers. There have been high resolution microwave ${ }^{17}$ and infrared $^{18}$ studies on acetaldehyde confirming the barrier of $408 \mathrm{~cm}^{-1}$ and the equilibrium structure as eclipsed conformer (figure 2). Thus the acetaldehyde barrier is approximately one third than that in ethane. This fact would appear to fit Pauli repulsion model ${ }^{11}$ since there is one $\mathrm{CH}-\mathrm{CH}$ eclipsed interaction in acetaldehyde staggered geometry (figure 2) whereas in ethane there are three. But this explanation could not answer why the barrier in propene $^{19}\left(\sim 700 \mathrm{~cm}^{-1}\right)$ is greater than that in acetaldehyde even though there is one $\mathrm{CH}-$ $\mathrm{CH}$ eclipsed interaction (figure 2). Although, acetaldehyde has been singled out because of its large $\mathrm{C}=\mathrm{O}$ bond dipole causing polarization effects on the methyl $\mathrm{C}-\mathrm{H}$ bonds ${ }^{20}$ and because of its oxygen lone pair electrons leading to a possible weak covalent bond between the carbonyl oxygen and an in-plane methyl hydrogen. ${ }^{21}$ But the dipole polarization mechanism predicts that the acetaldehyde barrier, driven by the large $\mathrm{C}=\mathrm{O}$ dipole, should be increased over that in propene ${ }^{20}$ - contrary to what is observed. Further no critical point is found between oxygen and hydrogen nuclei. ${ }^{22,23}$

Though, obviously, the explanation of the origin of barrier changes from molecule to molecule, a model is always advantageous to predict the barrier and its cause in a given series of molecules. In the mid seventies Hehre, Pople and Devaquet, ${ }^{24}$ using the split valence shell molecular orbital theory, first proposed a model which could qualitatively predict the barriers in many methyl conjugated molecules. This model is very useful in the sense that the barrier and the stabilization of the methyl rotor conformation could be understood by considering only the interactions between the fragmented orbitals, the

(a)

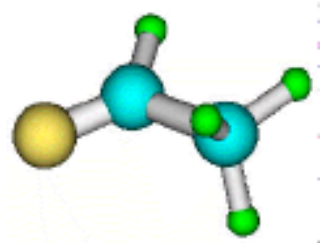

Staggered

(b)

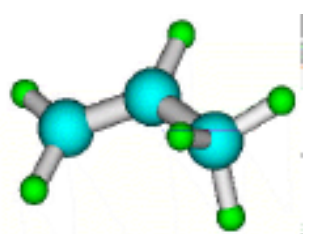

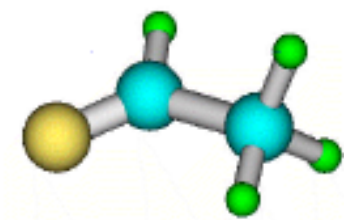

Eclipsed

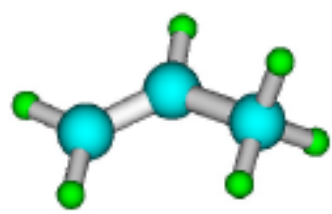

Figure 2. Staggered (at the top of barrier) and eclipsed (preferred) conformers of (a) acetaldehyde and (b) propene. 
valence orbital of the methyl group ${ }^{25}$ and the out-of-plane $\pi$ orbital of vicinal unsaturated linkage such as $\mathrm{C}=\mathrm{C}$ in case of propene.

The barrier results from four interactions between the highest occupied and lowest vacant molecular orbitals on each of two fragments. The relative positions of these fragmented orbitals are shown in the figure 3. Figure 4 shows plot of these fragmented orbitals for propene molecule. Interaction 1 is the repulsion between filled $\pi$-like methyl group orbitals $\left(\pi_{\text {methyl }}\right)$ and filled $\pi$ orbital of the double bond $\left(\pi_{\text {louble-bond }}\right)$. As seen in figure 4 , the orbital overlap is greater in staggered conformation than that in eclipsed one.

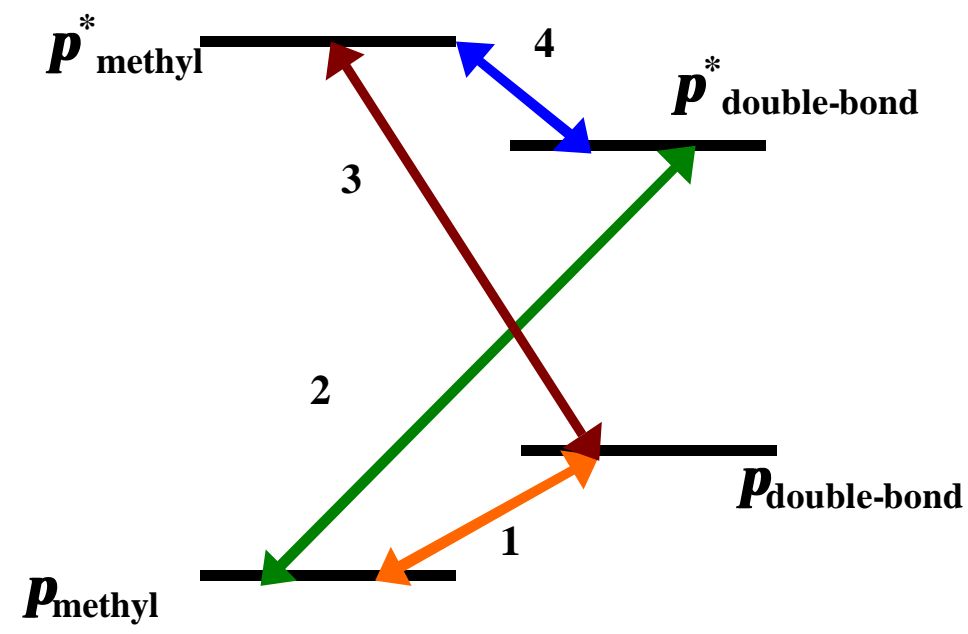

Figure 3. Relative position of valence orbitals of the methyl group and the $\pi$-orbitals of the double bond. 1, 2, 3, 4, represent different interactions between them.
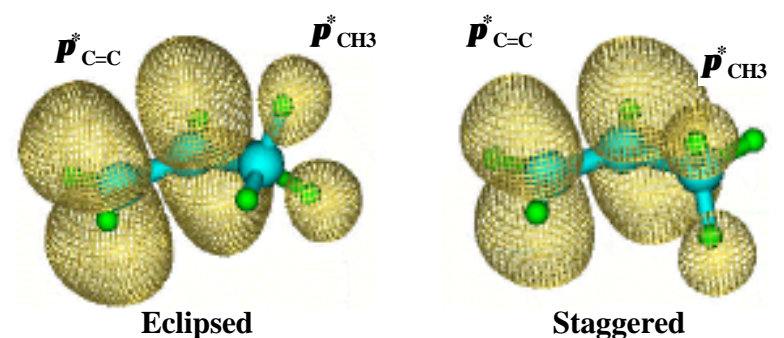

$$
\text { Staggered }
$$
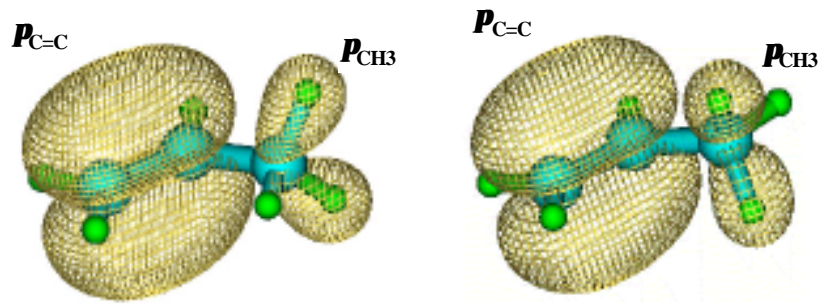

Figure 4. Plot of fragmented orbitals $\left(\pi_{\text {methyl }}, \pi_{\mathrm{C}=\mathrm{C}}, \pi^{*}{ }_{\text {methyl }}, \pi^{*}{ }_{\mathrm{C}=\mathrm{C}}\right)$ for propene in eclipsed and staggered conformation. 
Since the interaction energy is proportional to the square of the overlap between these orbitals, the repulsion is increased in the staggered conformation. Thus the energy for this geometry is greater than the eclipsed one. Interaction 2 is between $\pi_{\text {methyl }}$ and $\pi^{*}$ double-bond whereas interaction 3 is between $\pi^{*}{ }_{\text {methyl }}$ and $\pi_{\text {louble-bond. }}$. Both of these interactions are stabilizing because they involve only two electrons as opposed to the 4-electron repulsion arising from filled $\pi$ fragment orbitals in interaction 1 . Here also eclipsed conformation is preferred because in staggered geometry the overlap between orbitals is significant but of opposite sign. Interaction 4 in case of ground state of neutral molecule is not relevant. Thus the result of these three interactions is that the eclipsed conformer is preferred over the staggered one. This model has been extended to radical cations, anions and triplet states by Dorigo, Pratt and Houk $^{26}$ taking into account the interaction between $\pi^{*}$ methyl and $\pi^{*}$ double-bond as well.

The discussion above is based on propene where the double bond is non-polar. The next point is how the barrier changes when the polarity of the double bond either increases or decreases compared to $\mathrm{C}=\mathrm{C}$ in propene. If one of the carbon atoms in this unsaturated bond is replaced by an atom with greater electronegativity, the $\pi$ and $\pi^{*}$ orbital energies are lowered, decreasing the energy separation between $\pi_{\text {methyl }}$ and $\pi^{*}$ double-bond and increasing that between $\pi^{*}$ methyl and $\pi_{\text {louble-bond. }}$. The interaction between filled orbitals (interaction 1) is independent of separation of energy and hence remains unchanged. Here we restrict our discussion only to acetaldehyde where far carbon atom is replaced by more electronegative oxygen atom. As a result of this replacement the $\pi$ fragmented orbital of this bond gets more localized at the oxygen for the filled $\pi$ orbital whilst the reverse is true for the unfilled $\pi^{*}$ orbital. A comparison of this localization between $\mathrm{C}=\mathrm{O}$ and $\mathrm{C}=\mathrm{C}$ is shown in figure 5. Keeping in mind the energy shift due to polar double bond, the interaction 2 in this case increases and conformational preference of eclipsed structure reduces. At the same time, due to the localization effect the overlap in the staggered conformation decreases (figure 5) interaction 3, however, is small due to

(a)
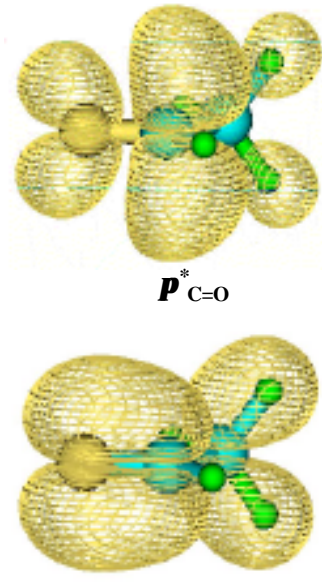

$\boldsymbol{\pi}_{\mathrm{C}=\mathrm{O}}$ (b)

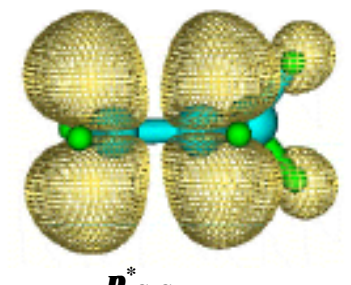

$\pi^{*}=\mathrm{C}$

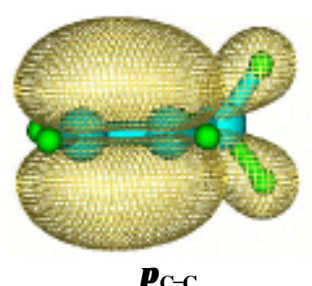

$\pi_{\mathrm{C}=\mathrm{C}}$

Figure 5. Plot of fragmented orbitals $\pi$ and $\pi^{*}$ for $(\mathbf{a})$ acetaldehyde $(\mathrm{C}=\mathrm{O})$ and (b) propene $(\mathrm{C}=\mathrm{C})$. In $\mathrm{C}=\mathrm{O}$, the orbital is more localised on oxygen in $\pi$ and on $\mathrm{C}$ in $\pi^{*}$. 
the increase in energy separation although the overlap increases due to localization effect. The overall effect of all these interactions is to reduce the barrier height and thus acetaldehyde has lower barrier than propene. Extrapolation of this model predicts that the increased polarity due to the replacement of more electronegative atom at the far position of the double bond decreases the barrier.

Even ignoring the interactions within $\sigma$ framework this model has been successful to guess the preferred methyl group conformation and qualitatively the barrier height for many methyl conjugated molecules but deeper insight into the barrier is surprisingly different. In the following we will discuss how the $\sigma$ electrons play an important role on barrier formation along with $\pi$ electrons. ${ }^{22,27,28}$

As discussed in $\$ 2$ the most primitive approach to internal rotation is the rigid rotation path where only the torsional angle $(\tau)$ is allowed to vary keeping all other skeletal degrees of freedom frozen. This decouples the torsion with any other local mode vibrations present in the molecule. By this simple model, ab initio calculated barrier is only $\sim 10 \%$ above the experimental barrier (see table 1).

The potential surface is the adiabatic fully relaxed (FR) pathway with respect to all other coordinates, hence cannot be truly one dimensional. Ozkabak and Goodman ${ }^{8 c}$ pointed out that the internal rotation is a multidimensional process and addressed the skeletal flexing of the molecule accompanying the rotation. A major conclusion is that the rigid rotation does not provide the threefold periodic potential. Also, lengthening of $\mathrm{C}-\mathrm{C}$ bond in the methyl conjugated molecules is an important factor for barrier determination. Subsequent studies showed that the key to the potential shape near the bottom of the well is provided by aldehyde hydrogen flexing in acetaldehyde. ${ }^{29}$ Goodman, Leszczynski and $\mathrm{Kundu}^{30}$ showed that methyl torsion initiates ethylenic $\left(-\mathrm{CH}_{2}\right)$ twisting and out of plane wagging of the hydrogen adjacent to the methyl group in propene as well. Their important deduction was that these motions, intrinsic parts of the torsional coordinate, cannot be neglected in understanding the shape of potential barrier. Thus, the picture of internal rotation is that of highly impure vibration comprising large amplitude motion coupled to skeletal normal modes.

In view of this, when the multidimensional nature is incorporated relaxing all the degrees of freedom (fully relaxed), the outcome is nearly correct barrier with an accurate treatment of electron correlation (table 1). The small corrections of $20-30 \mathrm{~cm}^{-1}$ in barrier energy from previous calculation may lead to delusion that barrier could be understood by rigid rotation. Detailed studies showed that the generalizations are not meaningful in this approach because multidimensional corrections to partitioned attractive and repulsive energies are very large and even change the sign of individual energy components. In the following we will critically scrutinize the origin of potential barrier by partitioning the barrier energy as discussed in $§ 2$.

Table 1. MP2/6-311G(3df, $2 p)$ ab initio torsional barriers $\left(\mathrm{cm}^{-1}\right)$.

$[\Delta E=$ staggered-eclipsed $]$ for acetaldehyde and propene

\begin{tabular}{lcccc}
\hline & & \multicolumn{3}{c}{ Fully relaxed } \\
& Expt. & Rigid frame & frame & Correction \\
\hline Acetaldehyde & $408^{\mathrm{a}}$ & 475 & 438 & 37 \\
Propene & $693^{\mathrm{b}}$ & 743 & 719 & 24 \\
\hline
\end{tabular}

${ }^{\mathrm{a}}$ Ref. 17,$18 ;{ }^{\mathrm{b}}$ Ref. 19 
Table 2. Ab initio fully relaxed calculated partitioned barrier energetics $\left(\mathrm{cm}^{-1}\right)$ in propene.

\begin{tabular}{lcc}
\hline Energy difference & $\mathrm{HF} / 6-31 \mathrm{G}(d, p)$ & $\mathrm{MP} 2 / 6-311 \mathrm{G}(3 d f, 2 p)$ \\
\hline Total energy $(\Delta E)$ & 725 & 719 \\
Kinetic energy $(\Delta T)$ & -837 & -654 \\
Potential energy $(\Delta V)$ & 1562 & 1373 \\
$\quad$ Nuclear-nuclear & & \\
$\quad$ Repulsion energy $\left(\Delta V_{n n}\right)$ & -28424 & -30877 \\
$\quad$ Electron-electron & & \\
$\quad$ Repulsion energy $\left(\Delta V_{e e}\right)$ & -23172 & -26785 \\
$\quad$ Nuclear-electron & & \\
$\quad$ Attraction energy $\left(\Delta V_{n e}=\Delta V_{a}\right)$ & 53157 & 59034 \\
$\quad$ Repulsion energy $\left(\Delta V_{r}=\Delta V_{n n}+\Delta V_{e e}\right)$ & -51595 & -57662 \\
\hline
\end{tabular}

3.2a Potential type: Total energy difference (barrier) and partitioned energetics in propene molecule for fully relaxed method are listed in table 2 for two calculation levels: $\mathrm{MP} 2 / 6-311 \mathrm{G}(3 d f, 2 p)$ and $\mathrm{HF} / 6-31 \mathrm{G}(d, p)$. The comparison between these levels of calculation shows that even with modest basis set $[6-31 \mathrm{G}(d, p)]$ varitaional Hartree-Fock (HF) method predicts a reasonable barrier with correct sign of individual energetics to describe the physics of the barrier. The reason for introducing HF is that it satisfies variational principle. This is to be noted that although there are large quantitative difference between HF and higher level (MP2) partitioned energetics, all calculation levels yield the same qualitative outcome; an increase in potential energy, despite decrease in overall electron and nuclear repulsion energies on the top of the barrier. The increase in potential energy stems from the enhanced attraction $\left(\Delta V_{n e}\right)$. At the same time kinetic energy decreases. Figure 6 shows the formation of the barrier from individual energies in fully relaxed model. The energetics for the one-dimensional rigid rotation process is also compared with fully relaxed one in this figure. The total energy change lies modestly above the true barrier $\left(719 \mathrm{~cm}^{-1}\right)$. The change in individual energy terms are much more significant. The rigid rotation increases the kinetic energy but decreases the potential energy despite the increased repulsion. This decrease results from decrease in electron-nuclear attraction energies. Since the kinetic energy increases more strongly than the decrement of potential energy, there is an overall raising of the staggered conformation energy above eclipsed one. This picture is completely reversed for fully relaxed model.

These partitioned energetics for acetaldehyde are listed in the table 3. Although the individual magnitudes are quite different, the overall picture remains same for this molecule also. The dilemma between these two approaches (rigid and fully relaxed) remains unresolved until we understand how the skeletal relaxation changes the picture going from rigid rotation to fully relaxed model.

3.2b Nuclear virial type: For non equilibrium conformation (rigid frame) the contribution of nuclei to the virial of the forces acting on the electrons do not vanish. Since the rigid rotation barrier is the difference between a state where the forces do not vanish and the equilibrium state where they do, the difference represents only nuclear 
Table 3. $A b$ initio calculated MP2/6-311G(3df,2p) partitioned energy difference $\left(\mathrm{cm}^{-1}\right)$ for acetaldehyde internal rotation path.

\begin{tabular}{lcc}
\hline Energy difference & Rigid frame & Fully relaxed \\
\hline Total energy $(\Delta E)$ & 475 & 438 \\
Kinetic energy $(\Delta T)$ & 1395 & -176 \\
Potential energy $(\Delta V)$ & -920 & 614 \\
Nuclear-nuclear & & \\
$\quad$ Repulsion energy $\left(\Delta V_{n n}\right)$ & 2771 & -5822 \\
$\quad$ Electron-electron & & \\
$\quad$ Repulsion energy $\left(\Delta V_{e e}\right)$ & 3847 & -3313 \\
Total repulsion energy $\left(\Delta V_{r}\right)$ & 6618 & -9135 \\
Nuclear-electron & & \\
$\quad$ Attraction energy $\left(\Delta V_{n e}\right)$ & -7539 & 9749 \\
\hline
\end{tabular}

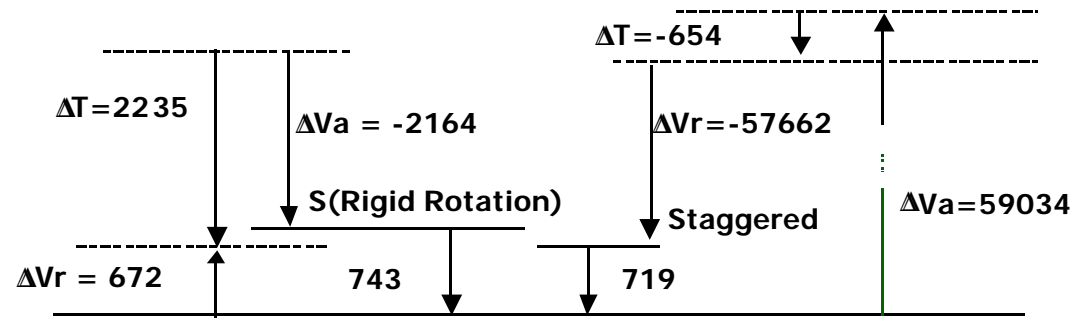

Equilibrium (eclipsed)

Figure 6. Formation of internal rotation barrier from individual repulsive, attractive and kinetic energies for rigid frame and fully relaxed model in propene.

virial for the nonequilibrium state. Thus according to virial theorem, ${ }^{31,32}$ for the rigid rotation process,

$$
\Delta T=-\Delta E+\sum_{\alpha} \chi_{\alpha} \cdot F_{\alpha} .
$$

Thus the potential energy change of the electrons is dominated by the virial repulsive forces acting on the nuclei, when the acetaldehyde skeleton is frozen during methyl torsion. Figure 7 shows the calculated $[\mathrm{HF} / 6-31 \mathrm{G}(d, p)]$ nuclear virials in acetaldehyde for different skeletal relaxations. ${ }^{33}$ The six fold virial originates from the out-of-plane wagging motion of aldehyde hydrogen, the threefold one is due to lengthening of $\mathrm{C}-\mathrm{C}$ bond and the combination of one and two fold virial is due to other skeletal flexing such as change in methyl top, rotation axis etc. To understand the effect of these skeletal flexings on individual barrier energetics, the entire rotational processes can be divided into three consecutive conceptual paths. 


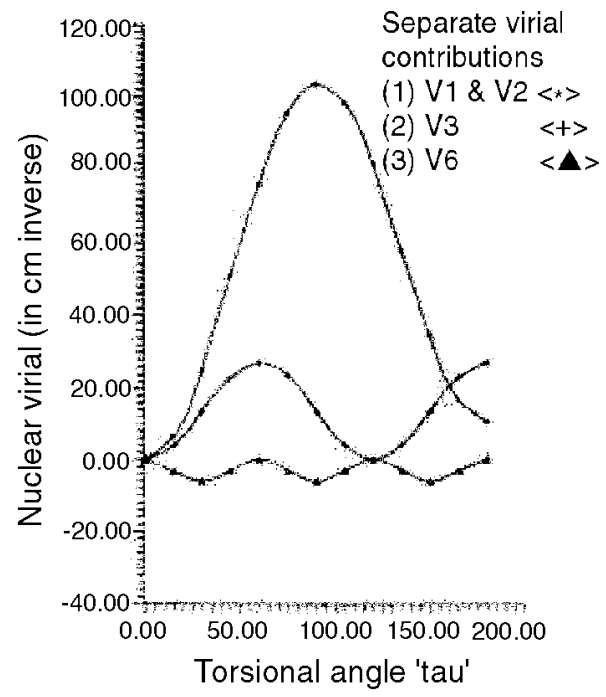

Figure 7. Calculated nuclear virials for individual relaxation in acetaldehyde. Sixfold $\left(V_{6}\right)$ for aldehyde hydrogen flexing, threefold $\left(V_{3}\right)$ for $\mathrm{C}-\mathrm{C}$ bond lengthening and others $\left(V_{1}, V_{2}\right)$ for other flexings.

Path I: Rigid rotation where the molecule is fixed at the eclipsed conformer, except that the methyl group is rotated by $60^{\circ}$ to its staggered position.

Path II: The $\mathrm{C}_{\text {methyl }}-\mathrm{C}$ bond is lengthened to its value in the fully relaxed conformer.

Path III: All other flexings required to reach the fully relaxed geometry are allowed to relax.

It is to be noted that the aldehyde hydrogen remains in plane at the eclipsed and staggered geometry, so that the nuclear virial for this motion affects only the shape of the potential. The calculated energies for the above mentioned paths in propene are given in table 4. In the path II where rigid rotation (path I) is relaxed by lengthening of $\mathrm{C}-\mathrm{C}$ bond only, the large changes in individual energy terms and sign show the importance of this process. This is effectively hidden under the change of total energy difference $\left(14 \mathrm{~cm}^{-1}\right)$. The important outcome of this step is that the nuclear virial for this step is negative and overwhelms the positive nuclear virial of path I. The sign of the potential energy term $\Delta V$ changes from negative to positive and forms the barrier. The change in sign comes from an increase in $\Delta V_{n e}$ due to $\mathrm{C}-\mathrm{C}$ bond lengthening which overwhelms the decrease in the repulsive terms $\Delta V_{e e}$ and $\Delta V_{n n}$. Thus the energetics for path I + II are quantitatively the same as for the fully relaxed process. The magnitudes are different because of the negative nuclear virial residue for the incomplete internal rotation process defined by path I and path II. The remaining skeletal flexing modulate the large negative nuclear virial induced in path II. The effect of the path III is to stabilize the molecule possessing the stretched $\mathrm{C}-\mathrm{C}$ bond of the path II, keeping the physics of the process unchanged. This multidimensional rotation picture and importance of $\mathrm{C}-\mathrm{C}$ bond lengthening is also found true in acetaldehyde. The overview of these pathwise calculations is that although the rigid rotation barrier energy is not very much different from fully relaxed one, conclusions about the physics of barrier formation drawn from rigid rotation could be misleading. 
Table 4. Ab initio $\mathrm{HF} / 6-31 \mathrm{G}(d, p)$ partitioned energy difference $\left(\mathrm{cm}^{-1}\right)$ for propene internal rotation path.

\begin{tabular}{lrrr}
\hline Energy difference & Path I $^{\mathrm{a}}$ & Path II $^{\mathrm{a}}$ & Path III $^{\mathrm{a}}$ \\
\hline Total energy $(\Delta E)$ & 751 & 737 & 725 \\
Kinetic energy $(\Delta T)$ & 2122 & -1771 & -837 \\
Potential energy $(\Delta V)$ & -1371 & 2508 & 1562 \\
Nuclear-nuclear & & & \\
$\quad$ Repulsion energy $\left(\Delta V_{n n}\right)$ & -492 & -31204 & -28424 \\
$\quad$ Electron-electron & & & \\
$\quad$ Repulsion energy $\left(\Delta V_{e e}\right)$ & 3983 & -25929 & -23172 \\
$\quad$ Repulsion energy $\left(\Delta V_{r}\right)$ & 3491 & -57133 & -51596 \\
$\quad$ Nuclear-electron & & & \\
$\quad$ Attraction energy $\left(\Delta V_{n e}\right)$ & -4863 & 59640 & 53157 \\
\hline
\end{tabular}

${ }^{\mathrm{a}}$ For the description of these paths, see $\S 3.2 \mathrm{~b}$

Table 5. Symmetry type partitioned energies in $\mathrm{cm}^{-1}[\mathrm{HF} / 6-31 \mathrm{G}$ $(d, p)]$ for propene for different rotational path ${ }^{\mathrm{a}}$.

\begin{tabular}{llrrr}
\hline Energy difference & \multicolumn{1}{c}{ Symmetry } & Path I & Path II & Path III \\
\hline Kinetic energy $(\Delta T)$ & $\mathrm{A}^{\prime}(\sigma)$ & -1266 & -4968 & -4222 \\
& $\mathrm{~A}^{\prime \prime}(\pi)$ & 3388 & 3197 & 3385 \\
Total $(\Delta T)$ & $\mathrm{A}^{\prime}(\sigma)+\mathrm{A}^{\prime \prime}(\pi)$ & 2122 & -1771 & -837 \\
Nuclear-electron & & & & \\
$\quad$ Attraction energy & $\mathrm{A}^{\prime}(\sigma)$ & 36507 & 91660 & 86442 \\
$\quad\left(\Delta V_{n e}\right)$ & $\mathrm{A}^{\prime \prime}(\pi)$ & -41370 & -32020 & -33285 \\
Total $\left(\Delta V_{n e}\right)$ & $\mathrm{A}^{\prime}(\sigma)+\mathrm{A}^{\prime \prime}(\pi)$ & -4863 & 59640 & 53157 \\
\hline
\end{tabular}

${ }^{\mathrm{a}}$ For the description see $\$ 3.2 \mathrm{c}$

3.2c Symmetry type: The cause of $\mathrm{C}-\mathrm{C}$ bond relaxation, which leads to an interchange of $\Delta T$ and $\Delta V$ is an important key to deeper insight into the internal rotation processes for these methyl conjugated molecules. Here we focus to an important subdivision of overall energy partitioning. Since both the molecules acetaldehyde and propene, retain its $C_{\mathrm{S}}$ symmetry in the eclipsed and staggered conformations, it allows the potential term to be additionally broken down into contributions from $a^{\prime}$ and $a^{\prime \prime}$ orbitals. Since the molecular skeletons remain planar and methyl group valence orbitals can be classified as $\pi$ and $\sigma$ types, ${ }^{25}$ this subdivision allows one to separate the effects due to $\pi$ and $\sigma$ electrons. These sub-partitioned energies for propene are given in the table 5 at $\mathrm{HF} / 6-31 \mathrm{G}(d, p)$ level of the theory for three different paths mentioned in the earlier section. Table 5 shows that $\Delta T(\sigma)$ always decreases but $\Delta T(\pi)$ increases going from equilibrium eclipsed to staggered conformation at the top of the barrier. The increase in $\Delta T(\pi)$ is due to the increased $\pi$ electron overlap in the staggered conformation. Since in path I, $\Delta T$ is the barrier forming term the dominant contribution from $\pi$ electrons is the key for $\pi$ fragment model discussed earlier. The important observation is the change in sign $\Delta T$ and $\Delta V$ in path II or path III due to the enormous increase in $\sigma$ electrons contribution. So it is finally the $\sigma$ term that controls the barrier in actual internal rotation. 
The principal outcome of all these studies is that repulsive nature of $\pi$ electrons at the top of the barrier forces the molecule to follow the adiabatic path through skeletal flexings. In this path, the $\mathrm{C}-\mathrm{C}$ bond relaxation plays the dominant role in the barrier formation through the increased contribution in the energy from $\sigma$ electrons.

\subsection{Methyl torsion in aromatic molecules}

The torsional motion of methyl group is easily subject to a change in intramolecular interactions because this rotation is greatly affected by the environment of a group or a part in the molecule. The potential of this motion therefore reflects such interactions and gives a good measure of the molecular structure and dynamics. Toluene is the basic molecule in this category. Since it has sixfold symmetry, the threefold barrier should not arise in this molecule, contrary to experimental observation. The barrier in the electronic ground state is $\sim 10 \mathrm{~cm}^{-1} .{ }^{34-36}$ The origin of this barrier is attributed to the difference in $\pi$ bond order. ${ }^{37-39}$ Since the barrier for this methyl torsion depends on local bond order, it can be tailored by attaching different electron withdrawing or electron donating group in the benzene ring. ${ }^{40-45}$ There have been many high resolution studies of methyl torsional barrier for different aromatic molecules such as methyl indole, ${ }^{46}$ methyl aniline, ${ }^{47,48}$ acetophenone, ${ }^{49}$ methyl stilbene, ${ }^{50}$ cresol $^{51}$ methyl pyrimidine. ${ }^{52}$ The methyl torsional barrier in the aromatic molecules originates due to the difference in the $\pi$-bond order between two ring $\mathrm{C}-\mathrm{C}$ bonds nearest to methyl group. Liljefers and Allinger ${ }^{37}$ proposed that greater the $\pi$-bond order, lower the energy for eclipsed conformation than the staggered one. This idea has been extended by George et al $^{38}$ for toluene and several aromatic hydrocarbons. Lu et $a l^{39}$ proposed that if the steric effect is absent, the barrier height is proportional to the calculated bond order with a slope of $950 \mathrm{~cm}^{-1}$ per bond. Nakai et al $^{53}$ showed that the inplane $\mathrm{C}-\mathrm{H}$ eclipses the higher order $\mathrm{C}-\mathrm{C}$ bond due to the attractive donor-acceptor interaction between them. Variation of potential barrier in substituted toluenes such as fluorotoluene, cresol etc. is theoretically investigated by them and proposed a new mechanism involving $\pi^{*}$ and $\sigma^{*}$ hyperconjugation in the lowest unoccupied molecular orbital in these molecules. Taking this effect into account, the change in barrier could be rationalized for different substitutions.

Nitrogen containing heterocycles such as imino compounds are of central importance in diverse areas of biochemical processes and industrial applications. We have recently focused $^{54}$ on 1-methyl-2(1H)-pyridinimine molecule in an attempt to examine vibronic structure in the excited states. This molecule has another importance in understanding the origin of methyl torsional potential barrier because of the presence of nitrogen lone pair. ${ }^{55}$ It is observed that the high electron density of this lone pair influences the methyl configuration. Here the energy of staggered geometry is lesser than the eclipsed one. Figure 8 shows the electron density plot of the molecule in eclipsed conformation. In this configuration the repulsion between filled inplane $\mathrm{C}-\mathrm{H}$ and lone pair of nitrogen is greater than the repulsion of $\pi$ electrons in staggered conformation. This culminates in the barrier to methyl rotation and stabilizes the staggered conformation. The importance of this study is that the conformer of methyl group could be changed from staggered to eclipsed by tailoring the barrier. In some molecules, this change in methyl conformation may have far reaching implications for microscopic properties. 
<smiles></smiles>

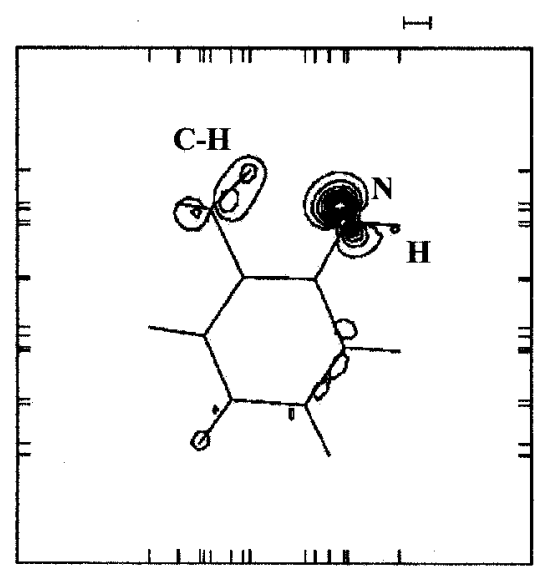

Figure 8. Nitrogen lone pair and filled $\mathrm{C}-\mathrm{H}$ orbitals interaction in eclipsed configuration of 1-methyl 2-(1H)-pyridinimine.

\section{Overview}

In this paper, we have attempted to provide a modest coverage of the ideas on methyl internal rotation barrier. Ethane, a well-studied molecule, is still in focus for its simplicity in unraveling the nuances of rotational barrier. Although, the steric repulsion is the spontaneous answer for its preferred structure, in depth analysis shows that hyperconjugation is also an important term in this regard and in fact, it forms the barrier to this rotation. The description of rotational dynamics in one dimension may provide a qualitative picture, but physics cannot be understood without including relaxation of the molecule through skeletal flexing. For example, in ethane, $\mathrm{C}-\mathrm{C}$ bond expansion during rotation plays an important role in balancing the individual energetics. This multidimensional description provides an adiabatic pathway for this motion and is successful in predicting torsional frequencies in many molecules. In search of the barrier origin for methyl conjugated molecules, the decomposition of total barrier energy into potential type, virial type and symmetry type reveals the importance of $\mathrm{C}_{\text {methyl }}-\mathrm{C}$ relaxation and discloses the contribution of $\sigma$ electrons. The compiled picture may be viewed as, during methyl rotation the nuclear virial repulsive energy appears from $\pi$ electrons. To remove the positive nuclear virial, the molecule has to relax by $\mathrm{C}-\mathrm{C}$ bond lengthening and due to this the $\sigma$ and $\pi$ core energies increase. The contributions from $\sigma$ electrons overwhelm that from $\pi$ electrons and forms the barrier to the rotation.

Although, the importance of $\sigma$ and $\pi$ electrons is addressed extensively, the literature on the effect of lone pair is sparse. We have recently recognised its importance in barrier formation and detailed studies in this direction are in progress. 
The impetus for this attention is that the height and shape of the barrier to internal rotation determine the dynamics and splitting brought about by torsional tunneling. The dynamical behaviour has important consequences to intramolecular vibrational relaxation and flexing behaviour in many organic and bio-organic molecules. Established role of inplane and out-of-plane electrons on barrier formation suggests that the methyl torsion has potential to become a probe for monitoring the local electronic environment of the molecule. Any perturbation of this environment such as in the Vander waal complexes or clusters could be monitored with this probe. The multidimensional nature of this motion could be exploited to understand the coupling of different large amplitude motions present in the molecule and the redistribution of energies in rovibrational levels.

\section{Acknowledgement}

We thank Prof L Goodman for valuable discussions and the Department of Science and Technology, Govt. of India for financial support.

\section{References}

1. Nielson H H 1932 Phys. Rev. 40445

2. Philis J G, Berman J M and Goodman L 1990 Chem. Phys. Lett. 167 16; (b) Kundu T, Thakur S N and Goodman L 1992 J. Chem. Phys. 97 5410; (c) Gu H, Kundu T and Goodman L 1993 J. Phys. Chem. 977194

3. Lister D G, MacDonald J N and Owen N L 1978 Internal rotation and inversion: An introduction to large amplitude motions in molecules (New York: Academic Press)

4. Reed A E and Weinhold F 1991 Isr. J. Chem. 31277

5. Payne W P and Allen L C 1977 In Application of electronic structure theory (ed.) H F Shaefer (New York: Plenum) p. 29

6. Villard A 1974 In Internal rotation in molecules (ed.) W J Orville-Thomas (New York: John Wiley) p. 385

7. Ito M 1994 Large-amplitude motions of aromatic molecules as studied by supersonic jet spectroscopy. Dynamics of excited molecules (ed.) K Kuchitsu (Amsterdam: Elsevier Science) ch 5, p. 97; (b) Spangler L H and Pratt D W 1995 In Jet spectroscopy and molecular dynamics (eds) J M Hollas and D Phillips (London: Chapman and Hall) p. 369

8. Swalen J D and Costein C C 1959 J. Chem. Phys. 31 1562; (b) Brant D M, Pugmire R J, Livingston R C, Strong K A, McMurry H L and Brugger R M 1970 J. Chem. Phys.52 4424; (c) Ozkabak A and Goodman L 1992 J. Chem. Phys. 965958

9. Kemp D and Pitzer K S 1936 J. Chem. Phys. 4 749; (b) Kemp D and Pitzer K S 1937 J. Am. Chem. Soc. 59 276; (c) Pitzer K S 1937 J. Chem. Phys. 5 466, 473

10. Pitzer R M 1951 Discuss. Faraday Soc. 1066

11. Sovers O J, Kern C W, Pitzer R M and Karplus M 1968 J. Chem. Phys. 492592

12. Gavezzotti A and Bertell I S 1979 J. Am. Chem. Soc. 1015142

13. Brunk T K and Weinhold F 1979 J. Am. Chem. Soc. 1011700

14. Bader R W F, Cheeseman J K, Laidig K E, Wiberg K B and Breneman C 1990 J.Am. Chem. Soc. 1126530

15. Goodman L, Gu H and Prophristic V 1999 J. Chem. Phys. 1104266

16. Prophristic V and Goodman L 2001 Nature (London) 411565

17. Belov S P, Yu Tretyakov M, Kleiner I and Hougan J T 1993 J. Mol. Spectrosc. 16061

18. Kleiner I, Hougan J T, Suenram R D, Lovas F J and Godefroid M 1991 J. Mol. Spectrosc. 148 38; (b) Kleiner I, Hougan J T, Suenram R D and Lovas F J 1992 J. Mol. Spectrosc. 153578

19. Hollenstein H and Winther F 1978 J. Mol. Spectrosc. 71 118; (b) Durig J R, Guirgis G and Bell S J 1989 J. Phys. Chem. 93348

20. Hadad C M, Foresman J B and Wiberg K B 1993 J. Phys. Chem. 974293

21. Jorgensen W L and Allen L C 1971 J. Am. Chem. Soc. 93 567; (b) Munoz-Caro, C, Nino A and Moule D C 1994 Theor. Chim. Acta 88299 
22. Goodman L, Kundu T and Leszczynski J 1995 J. Am. Chem. Soc. 1172082

23. Wiberg K B and Martini E 1985 J. Am. Chem. Soc. 1075035

24. Hehre W J, Pople J A and Devaquet J P 1976 J. Am. Chem. Soc. 98664

25. Hoffman R 1970 Pure Appl. Chem. 24567

26. Darigo A E, Pratt D W and Houk K N 1987 J. Am. Chem. Soc. 1096591

27. Kundu T, Goodman L and Leszczynski J 1995 J. Chem. Phys. 1031523

28. Goodman L, Kundu T and Leszczynski J 1996 J. Phys. Chem. 1002770

29. Goodman L, Leszczynski J and Kundu T 1994 J. Chem. Phys. 100 1274; (b) Nino A, MunozCaro C and Moule D C 1994 J. Phys. Chem. 981519

30. Goodman L, Leszczynski J and Kundu T 1993 J. Am. Chem. Soc. 11511991

31. Slater J C 1933 J. Chem. Phys. 1687

32. Nelander B 1969 J. Chem. Phys. 51469

33. Mitra A 1995 Study of methyl torsional potential surface in acetaldehyde M Sc dissertation, Indian Inst. Technol., Bombay, Mumbai

34. Walker R, Richard E C and Weisshaar J C 1996 J. Phys. Chem. 1007333

35. Eas A L L, Liu H, Lim E C, Jensen P, Dechene I, Zgierski M Z, Siebrand W and Bunker P R 2000 J. Chem. Phys. 112167

36. Breen P J, Warren J A, Bernstein E R and Selmen J I 1987 J. Chem. Phys. 87 1917; (b) Kreiner W A, Rudolph H D and Tan B T 1973 J. Mol. Spectrosc. 48 86; (c) Amir-Ebrahim V, Choplin A, Domaison J and Roussy G L 1981 J. Mol. Spectrosc. 66281

37. Liljefors T and Allinger N L 1985 J. Comput. Chem. 6478

38. George P, Bock C W, Stezowski J J, Hildenbrand T and Glusker J P 1988 J. Phys. Chem. 92 5656

39. Lu K T, Weinhold F and Weisshaar J C 1995 J. Chem. Phys. 1026787

40. Yam S and Spangler L H 1995 J. Chem. Phys. 993047

41. Siewert S S and Spangler L H 1995 J. Phys. Chem. 999316

42. (a) Takazawa K, Fuzzi M and Ito M 1993 J. Chem. Phys. 95 3205; (b) Okuyama K, Mikami N and Ito M 1985 J. Phys. Chem. 895617

43. Breen P J, Warren J A and Bernstein E R 1987 J. Am. Chem. Soc. 1093453

44. Walker R A, Richard E C, Lu K T and Weisshaar J C 1995 J. Phys. Chem. 9912422

45. Breen P J, Warren J, Bernstein E and Seeman J 1987 J. Chem. Phys. 871927

46. Sammeth D M, Siewert S S, Callis P R and Spangler L H 1992 J. Phys. Chem. 965771

47. Yam S and Spangler L H 1992 J. Chem. Phys. 964106

48. Disselkamp R, Lim H S and Bernstein E R 1992 J. Chem. Phys. 977889

49. Tomer J L, Spangeler L H and Pratt D N 1988 J. Am. Chem. Soc. 1101615

50. Metzger B S and Spangler L H 1997 J. Phys. Chem. 1015431

51. Mizuno H, Okuyama K, Ebata T and Ito M 1987 J. Phys. Chem. 915589

52. Bandy R, Nash J and Zwier T 1991 J. Chem. Phys. 952317

53. Nakai H and Kawai M 2000 J. Chem. Phys. 1132168

54. Pradhan B, Singh B P and Kundu T 2001 Proceedings of National laser symposium (Indore, CAT) p. 309

55. Pradhan B, Singh B P and Kundu T (in preparation) 